\title{
DARK MATTER IN THE UNIVERSE
}

\section{J. KORMENDY and G. R. KNAPP (EDS.)}

This is the first time that the International Astronomical Union has held a symposium on objects of totally unkown nature. In fact, it has been pointed out that the mass of the individual particles that make up the dark matter is unknown to $>70$ orders of magnitude. Since dark matter appears to make up $\sim 90 \%$ of the mass of the Universe, it presents us with one of the most fundamental problems in astrophysics.

The idea that the Universe might contain much more mass than can be seen in gas, stars and their remnants has been with us for over 50 years.

Until recently little more was known than that dark matter appears to exist; there was little systematic information about its properties. Only in the past several years was progress made to the point where dark matter density distributions can be measured. For example, with accurate rotation curves extending over large ranges in radius, decomposing the effects of visible and dark matter to measure dark matter density profiles can be tried. Some regularities in dark matter behaviour have already turned up.

This volume includes the 31 review and invited papers, 72 of the 85 poster papers, and the two general discussions. 